\title{
IL MOVIMENTO PROGRESSISTA IN AMERICA TRA RIFORME SOCIALI E MUTAMENTI COSTITUZIONALI
}

\author{
di Giuseppe Bottaro
}

All'inizio del ventesimo secolo, il movimento progressista ebbe il merito di riuscire ad ampliare la base sociale della democrazia americana, consolidando i meccanismi istituzionali di partecipazione e accrescendo il ruolo dell'opinione pubblica nel sostegno alle riforme

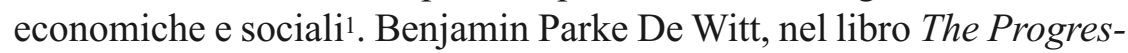
sive Movement, sostenne come nel progressismo statunitense fosse possibile individuare tre propensioni che lo caratterizzavano e lo distinguevano da tutte le correnti politico-culturali antecedenti: l'estensione delle funzioni del governo federale; l'ampliamento del controllo popolare sulle istituzioni democratiche; l'abolizione, o quanto meno il forte ridimensionamento, degli interessi particolari e particolaristici con le conseguenti valorizzazione e tutela dell'interesse generale del popolo americano ${ }^{2}$.

L'obiettivo fondamentale di questo movimento filosofico-politico era quello di rifondare il paese sopra solide basi democratiche, alla ricerca di prospettive più egualitarie. Per realizzare tutto ciò occorreva che il governo fosse al servizio dell'uomo e della comunità e non della proprietà. La fede nella ragione professata dai progressisti americani generava un'ottimistica visione del mondo in base alla quale il pro-

Dipartimento di Scienze Politiche e Giuridiche, Università di Messina.

1 Su queste tematiche vedi R. HOFSTADTER, L'età delle riforme: da W. Bryan a F.D. Roosevelt, Bologna, il Mulino, 1962. Cfr. anche A. TeSTI (a cura), L'età progressista negli Stati Uniti, 1896-1917, Bologna, il Mulino, 1984; J. KloppenBerg, Uncertain Victory. Social Democracy and Progressivism in European and American Thought, 1870-1920, New York, Oxford University Press, 1986.

2 Cfr. B.P. De WitT, The Progressive Movement, New York, Macmillan, 1915. 
gresso economico e sociale dell'America sarebbe stato inevitabile e avrebbe condotto a una società inclusiva, nella quale si sarebbero potuti armonizzare i tanti differenti interessi. I tratti distintivi del liberalismo progressista risultano essere, quindi, il ruolo positivo dello Stato, la supremazia del bene comune e l'inevitabilità del progresso3.

Un disegno comune a tutti gli esponenti del movimento progressista era di fare in modo che l'America tornasse ad essere un paese nel quale la realizzazione della democrazia sociale e il conseguimento dell'eguaglianza delle opportunità fossero i fini del sistema politico. Occorreva riconoscere alle istituzioni democratiche statali e federali un ruolo attivo e riformista nella garanzia dei diritti di libertà, di uguaglianza e d'indipendenza dei cittadini, contro le palesi ingiustizie presenti nella società americana ${ }^{4}$.

Si deve, pertanto, intendere il progressismo quale genuino impulso verso la critica della realtà sociale e verso gli inevitabili mutamenti che bisognava perseguire. "Il suo tema generale era la volontà di restaurare il tipo di individualismo economico e di democrazia politica che doveva esistere un tempo in America, prima che andasse distrutto per opera delle grandi società commerciali e della corruzione politica"s.

La gran parte dei progressisti pensava, tuttavia, che non fosse proponibile semplicemente ritornare agli Stati repubblicani di fine Settecento; il desiderio era di trasformare il liberalismo e il federalismo, vale a dire di farli diventare più conformi ai principi del progressismo e di adattarli alla repubblica statunitense del ventesimo secolo. De Caprariis descrive in maniera mirabile le modificazioni e i fermenti di questo periodo della storia americana. "Tra il 1870 e il $1910 \mathrm{gli}$ Stati Uniti si trasformano con una rapidità impressionante: 1'attività

3 Sul movimento progressista e sui profondi cambiamenti intervenuti in seguito alla sua affermazione nei primi decenni del Novecento, vedi A. ManN, The Progressive Era: Major Issues of Interpretation, Hinsdale, Dryden Press, 1975; A.S. LINK, R.L. McCormick, Progressivism, Arlington Heights, Harlan Davidson, 1983; J.W. Chambers, The Tyranny of Change. America in the Progressive Era, 1890-1920, New Brunswick, Rutgers University Press, 2000; L. Gould, America in the Progressive Era 1890-1914, Harlow, Pearson Education, 2001

4 Per le riforme politiche e economiche realizzate nell'età progressista, cfr. R. WIEBE, Businessmen and Reform: A Study of the Progressive Movement, Cambridge, Mass., Harvard University Press, 1962, L. GouLD, Reform and Regulation: American Politics from Roosevelt to Wilson, New York, Alfred A. Knopf, 1986.

5 R. HOFSTADTER, L'età delle riforme: da W. Bryan a F.D. Roosevelt, cit., p. 7. 
agricola passa decisamente in secondo piano, le ciminiere diventano la nota dominante del paesaggio americano, la popolazione cresce vertiginosamente grazie anche all'afflusso degli immigrati europei, i villaggi diventano città e le città metropoli. Con lo smarrimento del senso comunitario entra in crisi anche l'individuo, che si sente stretto tra organizzazioni tanto più grandi di lui, la corporation e la centrale sindacale, e che vede in queste organizzazioni limiti insormontabili alla sua assoluta libertà" 6 .

Il tema unificatore divenne la protesta contro questa trasformazione di fondo: le ingiustizie sociali, lo strapotere dei trust e gli attentati alla libertà individuale. Sopra queste basi gli intellettuali progressisti credettero di dover trovare delle soluzioni ottimali, delle riforme radicali, per risolvere i grandi scompensi sociali e politici che si erano determinati. Solo un sistema istituzionale basato sulla reale cooperazione tra gli Stati federati e lo Stato federale, con la supremazia di quest'ultimo, avrebbe potuto dare delle effettive risposte ai crescenti problemi derivanti dall'industrializzazione e dall'urbanizzazione.

\section{Progressismo e costituzionalismo}

L'inizio di questo consapevole mutamento prese avvio con la critica radicale alla Costituzione federale, che fu uno dei cavalli di battaglia del movimento progressista7. I federalisti e la Costituzione del 1787 avevano subito già nei primi anni della repubblica forti critiche da parte degli antifederalisti, quali, ad esempio, Thomas Jefferson o John Taylor, mentre a metà dell'Ottocento era arrivato l'attacco da parte di John C. Calhoun. Tutti costoro, però, difendevano la sovranità degli Stati e la regione economico-politica meridionale degli Stati Uniti8.

6 V. De CAPRARIIS, L'età progressista nel pensiero di Richard Hofstadter, introduzione a R. HofsTADTER, "L'età delle riforme: da W. Bryan a F.D. Roosevelt", Bologna, il Mulino, 1962. p. XV.

7 Sulla Costituzione federale del 1787, C. Rossiter, 1787: The Grand Convention, New York, Macmillan, 1966; F. McDonald, Novus Ordo Seclorum: The Intellectual Origins of the Constitution, Lawrence, University Press of Kansas, 1985, M. Kammen, The Origins of the American Constitution: A Documentary History, New York, Penguin, 1986.

8 Su queste tematiche cfr. N.E. CunNINGHAM, The Jeffersonian Republicans: the Formation of Party Organitation, 1789-1801, Chapel Hill, University of North Carolina Press, 
Gli storici e intellettuali progressisti di inizio Novecento, Woodrow Wilson, Charles Beard, Herbert Croly, e Vernon Parrington tenderanno, invece, a distinguere lo spirito democratico insito nella Dichiarazione d'Indipendenza dal carattere repubblicano e più conservatore che è possibile riscontrare nella Costituzione federale9. I progressisti prendevano atto che la Costituzione tutelava essenzialmente i grandi interessi finanziari e industriali, mentre le istanze democratiche che salivano dai ceti medi venivano sistematicamente frustrate. La Corte suprema, inoltre, appellandosi rigidamente alle norme costituzionali, bocciava sistematicamente tutte le leggi che tendessero ad un avanzamento sociale o alla promozione dei diritti positivi dei cittadini. In questi termini la discussione si incentrò sulla convenienza o meno di apportare modifiche sostanziali a un documento che a detta di molti progressisti impediva il normale funzionamento di una democrazia avanzata.

Wilson considerava la Carta fondamentale semplicemente non più adeguata per condurre gli Stati Uniti alla completa formazione di un sistema costituzionale moderno. Al futuro presidente degli Stati Uniti non interessavano tanto le motivazioni economiche di Hamilton o Madison, quanto gli impedimenti che essi avevano costruito sul cammino di una sintesi vitale ed essenziale tra il volere della comunità e il suo governo centrale ${ }^{10}$.

La Costituzione sostanziale non si era potuta a pieno affermare nella sua naturale evoluzione poiché si doveva restare ancorati alla Costituzione formale, la quale poteva andar bene alla fine del Settecento,

1957; N.K. RISJORD, The Old Republicans: Southern Conservatism in the Age of Jefferson, New York, Columbia University Press, 1965, e soprattutto H.J. STORING, The Complete Anti-Federalist, 7 voll., Chicago, University of Chicago Press, 1981. Più in generale sulle controversie politiche fra federalisti e antifederalisti nel periodo successivo all'entrata in vigore della Costituzione, cfr. L. BAnning, After the Constitution, Party Conflict in the New Republic, Belmont, Wentworth Publishing, 1989; S. Elkins, E. McKITRICK, The Age of Federalism, the Early American Republic, 1788-1800, New York, Oxford University Press, 1993.

9 Cfr. W. Wilson, Constitutional Government in the United States, New York, Columbia University Press, 1908; H. Croly, The Promise of American Life, New York, The Macmillan Company, 1909; C.A. BEARD, An Economic Interpretation of the Constitution of United States, New York, The Macmillan Company, 1913; V.L. PARRINGTON, Storia della cultura americana, Torino, Einaudi, 1969.

${ }^{10}$ Cfr. D. MARION, Alexander Hamilton and Woodrow Wilson on the Spirit and Form of a Responsible Republican Government, in "Review of Politics", vol. 42, 1980, pp. 309-328. 
ma non dava più risposte adeguate all'inizio del secolo americano. Il punto centrale dell'analisi diviene, pertanto, la teorizzazione dell'evoluzione del sistema costituzionale e l'affermazione, propria del movimento progressista, di una living constitution contrapposta alla corrente culturale conservatrice della original intention or interpretation ${ }^{11}$.

Gli scopi e gli sforzi principali della democrazia progressista, scrisse Croly nel 1914, dovrebbero essere indirizzati alla costruzione di una genuina comunità democratica. Occorreva instaurare un senso sempre più profondo nella costruzione di politiche e responsabilità comuni, così come maggiori interdipendenze fra gruppi e individui e una crescente cooperazione tra istanze democratiche e istituzioni nazionali ${ }^{12}$. La Costituzione federale doveva sempre più essere intesa come una living Constitution, che sarebbe potuta evolvere verso qualcosa non prevista o prevedibile rispetto a quella disegnata dai padri fondatori. Pertanto, "a living Constitution could be interpreted and reinterpreted to the point where the original principles were lost altogether. [...] the cure for the ills of the Founders 'republicanism was more centralized, national democracy"13.

Le battaglie intraprese dai progressisti per accrescere spazi e forme nuove di libertà, come le soluzioni prospettate per la risoluzione dei principali mali sociali, furono molteplici ${ }^{14}$. Si andava da coloro che volevano allargare lo spettro delle libertà economiche, a coloro che auspicavano un poderoso controllo statale sulle posizioni dominanti l'economia di mercato. Su altri versanti c'erano quelli che portavano avanti la lotta contro la segregazione razziale nel sud del paese o per l'inclusione sociale dei nuovi gruppi etnici giunti in seguito alle ondate migratorie, fino a coloro che si battevano per l'abolizione del lavoro

${ }^{11}$ Cfr. S. Pearson, Reinterpreting the Constitution for a New Era: Woodrow Wilson and the Liberal-Progressive Science of Politics, introduction to W. WILSON, "Constitutional Government in the United States", New Brunswick, Transaction Publishers, 2002, p. XXXIX.

${ }^{12}$ Cfr. H. Croly, Progressive Democracy, New Brunswick, Transaction Publishers, 2009 (originally published in New York, by The Macmillan Company, 1914), p. 238.

13 S. Pearson, JR., Herbert Croly and the Political Theory of Liberal Democracy, introduction to H. Croly, "Progressive Democracy", New Brunswick, Transaction Publishers, 2009, p. XXVIII.

${ }^{14}$ Cfr. A.S. Link, R.L. McCormick, Progressivism, Arlington Heights, Harlan Davidson, 1983; L. L. GouLD, Reform and Regulation: American Politics from Roosevelt to Wilson, New York, Alfred A. Knopf, 1986. 
minorile nelle fabbriche o che pensavano di instaurare un regime di proibizionismo sulla produzione e vendita degli alcolici ${ }^{15}$.

La relazione contraddittoria tra libertà politica e libertà industriale, o per meglio dire fra potere politico e potere industriale, era, secondo Brandeis, la vera questione irrisolta di quegli anni in America ${ }^{16}$. I lavoratori e le organizzazioni sindacali avrebbero dovuto imporre con maggiore fermezza il loro diritto all'autogoverno. Il ripristino di condizioni di libertà economica avrebbe permesso ai lavoratori di diventare cittadini attivi, membri di un'effettiva democrazia. La libertà del sistema industriale diviene, pertanto, la condizione indispensabile per consentire agli Stati Uniti, all'inizio del Novecento, di essere un autentico Stato federale, repubblicano e pluralista ${ }^{17}$.

Anche John Dewey, nel suo libro Comunità e potere, prova ad analizzare e a comprendere $\mathrm{i}$ grandi processi di trasformazione economica e sociale intercorsi nella storia recente degli Stati Uniti. All'inizio del Novecento in America "i rapporti quotidiani degli uomini avvengono in gran parte con grandi consorzi anonimi, con organizzazioni, non con altri individui. Questa non è altro, dunque, che una nuova era nei rapporti sociali, una nuova era nei rapporti umani" 18 . Democrazia per Dewey significa vivere in una libera e feconda comunione. Nondimeno lo sviluppo di società molto estese e fondate su un'avanzata forma di industrializzazione non consente relazioni dirette fra i cittadini e la nascita di una reale comunità. Se non vi è vita in comune, se non esiste un'idea di comunità non vi può essere democrazia ${ }^{19}$. La nuova libertà progressista non è più da intendersi come semplicemente negativa ma deve essere positiva. La legislazione e le istituzioni federali, derivanti dalle nuove norme costituzionali necessarie alla riforma della complessa società statunitense, devono intervenire per permettere all'individuo di essere libero dalle costrizioni imposte dalle corporazioni industriali. Dewey rende in maniera chiara ed evidente a tutti cosa significasse per lui e per progressisti quali Croly

${ }^{15}$ Cfr. H. FaulKner, Politics, Reform and Expansion, 1890-1900, New York, Harper \& Brothers, 1959.

${ }^{16}$ Cfr. L. Brandeis, Business. A Profession, Boston, Small, Maynard \& Co., 1914.

${ }_{17}$ Cfr. R.L. McCormick, The Party Period and Public Policy: American Politics from the Age of Jackson to the Progressive Era, New York, Oxford University Press, 1986.

18 J. Dewey, Comunità e potere, Firenze, La Nuova Italia, 1971, pp. 87-88.

19 J. DeweY, Comunità e potere, cit., p. 76. 
e Wilson questo concetto facendo riferimento all'idea di libertà effettiva. La promozione della libertà politica nel significato più ampio non può prescindere da una stretta relazione con le nuove istituzioni politiche riformate.

"La libertà è contemporaneamente un'idea e una pratica, un complesso di valori e un'esperienza che si attua attraverso il diritto e attraverso un insieme di politiche pubbliche. Il potere del governo è stato considerato da alcuni americani come un pericolo per la libertà, e da altri come uno strumento per raggiungere quella che John Dewey ha definito libertà effettiva - la capacità di dar vita alle istituzioni che determinano le caratteristiche della libertà" 20 .

\section{Progressismo ed elementi di democrazia diretta}

Le elezioni presidenziali statunitensi del 1912 rappresentarono un momento discriminante nella storia della repubblica federale americana. Il vero scontro elettorale si sviluppò tra l'ex Presidente Theodore Roosevelt e il candidato democratico Woodrow Wilson. Roosevelt, in seguito alla spaccatura ideologica verificatasi tra alcuni dei maggiori esponenti del partito repubblicano, decise di ricandidarsi alla testa del nuovo partito progressista, costituito dai maggiori esponenti dell'ala riformista dei repubblicani ${ }^{21}$.

La competizione elettorale si concentrò sui due candidati dotati di maggiore spessore politico, Roosevelt e Wilson, e sui loro programmi elettorali: New Nationalism e New Freedom ${ }^{22}$. I due programmi erano,

${ }^{20}$ E. Foner, Storia della libertà americana, Roma, Donzelli, 2000, p. 8. Vedi, anche, R.B. Westbrook, John Dewey and American Democracy, Ithaca, Cornell University Press, 1991.

${ }^{21}$ Questo importante periodo della storia statunitense è molto ben sviluppato da A. Schlesinger, The Rise of Modern America 1865-1951, New York, Macmillan, 1951, e J.L. Thomas, La nascita di una potenza mondiale: gli Stati Uniti dal 1877 al 1920, Bologna, il Mulino, 1988. Sul partito progressista si veda A. PINCHOT, History of the Progressive Party, 1912-1916, New York, New York University Press, 1958.

22 Per una comparazione fra queste due spiccate personalità di inizio ventesimo secolo si veda J.M. COOPER, The Warrior and the Priest: Woodrow Wilson and Theodore Roosevelt, Cambridge, Harvard University Press. 1983. Sulla figura di Roosevelt cfr. J.M. BLuM, The Republican Roosevelt, Cambridge, Harvard University Press, 1954, G. MowrY, The Era of Theodore Roosevelt and the Birth of Modern America, 1900-1912, New York, Harper, 1962; L. Gould, The Presidency of Theodore Roosevelt, Lawrence, University 
in effetti, molto simili e si fondavano largamente sulla lotta contro lo strapotere economico e politico dei grandi monopoli, i quali falsavano la libera concorrenza e penalizzavano i cittadini delle classi medie americane, come consumatori e come attori liberi della vita pubblica.

Nell'elaborazione del suo programma New Nationalism, Roosevelt si ispirò espressamente all'opera The Promise of American Life, scritta da Croly nel 190923. Il libro propugnava un più forte intervento dello Stato, l'avvento di uno spirito nazionalista che guidasse l'intero popolo americano e la realizzazione di molte delle riforme prospettate per un decennio dal movimento progressista: le elezioni primarie, il ricorso al referendum popolare, la legge sulla limitazione dell'orario di lavoro, e quelle sull'aumento delle retribuzioni minime per i lavoratori dipendenti, sulla tassazione progressiva dei redditi personali e sull'imposizione fiscale delle successioni24.

Il periodo delle grandi riforme a livello nazionale era stato preceduto, a livello dei singoli Stati, dalle battaglie politiche combattute e vinte fin dal 1900, l'anno di elezione del progressista Robert La Follette quale governatore del Wisconsin. "Fra il 1903 e il 1908 le primarie dirette entrarono negli statuti di 31 stati; ma non furono le uniche riforme di quegli anni. Nello stesso periodo, 12 stati passarono leggi di controllo

Press of Kansas, 1991. Sul pensiero e l'azione politica di Wilson si veda, fra gli altri A.S. LINK, Wilson, 5 voll., Princeton, Princeton University Press, 1947-1965; A.S. LINK, Woodrow Wilson and the Progressive Era: 1910-1917, New York, Harper \& Brothers, 1954; D. Steigerwald, The Synthetic Politics of Woodrow Wilson, in "Journal of History of Ideas", vol. 50, 1989, pp. 465-484; D. STID, The President as Statesman: Woodrow Wilson and the Constitution, Lawrence, University Press of Kansas, 2000; R.J. Pestritto, Woodrow Wilson and the Roots of Modern Liberalism, Lanham, Rowman \& Littlefield, 2005; G. BotTaro, Pace, libertà e leadership. Il pensiero politico di Woodrow Wilson, Soveria Mannelli, Rubbettino, 2007.

${ }^{23}$ Cfr. H. Croly, The Promise of American Life, New York, The Macmillan Company, 1909, B. DeXter, Herbert Croly and the Promise of American Life, in "Political Science Quarterly", vol. 70, 1955, p. 197. Nel 1914, insieme con Walter Lippmann e Walter Weyl, Croly fondò la rivista "The New Republic" che divenne ben presto l'organo ufficiale del movimento progressista. Cfr., D.W. NoBLE, The New Republic and the Idea of Progress, 19141920, in "The Mississippi Valley Historical Review", vol. 38, 1951, pp. 387-402, C. FoRCEY, The Crossroads of Liberalism. Croly, Weyl, Lippmann and the Progressive Era 1900-1925, New York, Oxford University Press, 1961, D. LEVY, Herbert Croly of the New Republic: The Life and Thought of an American Progressive, Princeton, Princeton University Press, 1985.

${ }^{24}$ Cfr. C.A. Beard, M.R. Beard, The Rise of American Civilization, 2 voll., New York, Macmillan, 1933; R. HOFSTADTER, L'età delle riforme: da Bryan a Roosevelt, Bologna, il Mulino, 1962; V.L. PARRINGTON, Storia della cultura americana, cit., vol. III. 
dell'attività delle lobbies, 22 posero dei limiti ai contributi delle corporation alle campagne elettorali, ben 41 introdussero forme di regolamentazione amministrativa delle ferrovie" 25 .

Wilson, nella New Freedom, evidenzia alcuni punti del suo programma elettorale che coincidono con le più importanti battaglie intraprese dal movimento progressista. Innanzitutto l'introduzione delle elezioni primarie, evento politico indispensabile per sviluppare un corretto rapporto tra eletti ed elettori e valido deterrente alla corruzione politico-elettorale. Occorre distinguere nettamente fra il leader, che dà forza ad un partito per condurlo verso il perseguimento del bene comune, e il boss, il quale normalmente si serve dello stesso partito per dirigere segretamente gli affari pubblici, occupare in maniera illecita parti consistenti dell'amministrazione e per compiere intrallazzi economici. Tutto ciò può essere superato, per Wilson, dalla scelta dei candidati attraverso le elezioni primarie. Questo sistema, infatti, consente agli iscritti ad un partito di scegliersi direttamente i politici che a loro giudizio sono dotati di maggiore leadership. Allo stesso modo egli esprime il suo pieno e convinto appoggio all'uso del referendum popolare. Non per abolire o limitare il sistema politico rappresentativo negli Stati Uniti, ma per responsabilizzare i legislatori. Questi, infatti, devono essere consapevoli che una loro disposizione legislativa contraria al bene comune potrà sempre essere sottoposta al voto popolare ed eventualmente abrogata ${ }^{26}$.

La definizione di questo programma e l'individuazione di questi obiettivi da realizzare avranno conseguenze rilevanti per la politica americana durante tutto il Novecento. Da questo momento in poi il partito democratico perderà il suo tradizionale ruolo di rappresentante politico della sezione Sud degli Stati Uniti per diventare sempre più il luogo di incontro delle istanze riformatrici e progressiste dell'intera federazione. Molti esponenti liberal abbandoneranno il partito repubblicano, così come l'idea di costituirne uno progressista, per confluire nel partito wilsoniano ${ }^{27}$.

25 A. Testi, L'età progressista negli Stati Uniti, 1896-1917, cit., pp. 51-52.

26 W. Wilson, The New Freedom: A Call for the Emancipation of the Generous Energies of a People, New York, 1913, ed. it. La nuova libertà. Invito di liberazione alle generose forze di un popolo, Milano, Istituto Editoriale Lombardo, 1914, pp. 155-157.

${ }^{27}$ Cfr. D. STID, The President as Statesman. Woodrow Wilson and the Constitution, cit. p. 112. 
"By 1916, when another presidential election would be held, Wilson had almost completely converted to supporting social legislation. The consequence was that many of the old Bull Moosers, people such as Jane Addams, the admired founder of Hull House in Chicago, John Dewey, the philosopher of pragmatism, Lincoln Steffens, the well-known muckraker, Walter Lippmann, the brillant young journalist, and, not to be forgotten, Herbert Croly himself, joined the Democratic party. Their support was of essential importance for a president in trouble, and for Democratic party, which received a clear progressive stamp"28.

Croly, nel suo libro Progressive Democracy del 1914, concepisce l'evoluzione dell'idea di democrazia americana come uno spostamento verso la partecipazione diretta dei cittadini nel processo di governo ${ }^{29}$. L'impulso del movimento progressista a sposare le tematiche contro la corruzione politica e di contrasto ai Trust industriali del precedente movimento populista, si accosta all'innovazione sul versante del ruolo dello Stato federale e dell'inevitabilità dello sviluppo industrial-capitalistico. Tutto ciò finisce col confluire nella tendenza ad introdurre nel sistema politico statunitense istituti quali l'elezione diretta dei senatori, passando attraverso le votazioni preliminari dirette (primarie), l'iniziativa legislativa popolare, il referendum, la destituzione degli eletti per voto popolare (recall). "Secondo il programma di Croly tutte queste misure dovevano contribuire all'istaurazione del governo diretto. Nel governo diretto il peso della volontà popolare doveva essere più forte, immediato e determinante, sostituendosi al potere delle assemblee rappresentative degli Stati e federali, nonché a quello, considerato pure troppo ligio alle concezioni economico-sociali dell'oligarchia plutocratica, delle Corti federali" 30 .

Allo stesso tempo, secondo Croly, occorreva aumentare i poteri del Presidente eletto dal popolo per far accrescere in maniera decisiva sia una nuova legislazione sociale sia una chiara pianificazione economica, azioni che avrebbero avuto come conseguenza il sensibile avanzamento delle condizioni di vita degli appartenenti alla classe operaia e alla classe media americana. Era, ormai, giunto il tempo di un confronto

28 J. W. Schulte Nordholt, Woodrow Wilson: A Life for World Peace, Berkeley, University of California Press, 1991, p. 98.

${ }^{29}$ H. Croly, Progressive Democracy, cit..

${ }^{30} \mathrm{O}$. BARIÉ (a cura), Il pensiero politico nell'età di Wilson, Bologna, il Mulino, 1961, p. 24. 
serrato tra democrazia rappresentativa e democrazia diretta. L'aspirazione verso un governo effettivamente popolare era stata bloccata dal sistema di pesi e contrappesi costituzionali per più di cent'anni. Altro fattore che impediva l'esercizio attivo da parte dei cittadini nel processo di governo era dovuto all'ignoranza politica del popolo americano. Se questi fattori fossero rimasti eguali anche nel ventesimo secolo sarebbe certamente bastata una qualche forma di democrazia rappresentativa. Invece, a giudizio di Croly, il contesto politico-sociale era mutato per sempre, così come erano cambiate le condizioni economiche che avevano favorito nel Settecento l'adozione di una forma di governo rappresentativo.

"In the twentieth century, however, these pratical conditions of political association have again changed, and have changed in a manner which enables the mass of the people to assume some immediate control of their political destinies. [...] The active citizenship of the country meets every morning and evening and discuss the affairs of the nation with the newspaper as an impersonal interlocutor. Public opinion has a thousand methods of seeking information and obtaining definite and effective expression which it did not have four generations ago"31.

In queste condizioni le discussioni che si sviluppano nel Congresso sono differenti rispetto al passato e hanno perso la funzione originaria di creazione e guida dell'opinione pubblica. I lavori delle Assemblee legislative si limitano a fornire uno specchio della stessa opinione pubblica, al massimo a proclamare e chiarire le idee e gli intenti che si formano altrove, nel popolo sovrano. Il rappresentante del popolo finisce con l'essere soltanto un "portavoce del popolo".

\section{La democrazia progressista}

Era giunto il tempo della democrazia progressista, occorreva sbarazzarsi delle vecchie formule del governo rappresentativo, del government by law o del government of law. Affermando ciò, Croly riteneva di demolire tutta la costruzione istituzionale elaborata da Madison, per arrivare a definire un nuovo meccanismo di governo diretto: una nuova

${ }^{31}$ H. Croly, Progressive Democracy, cit., pp. 263-264. 
genuina democrazia basata su nuove fasi di formazione, sviluppo e organizzazione dell'opinione pubblica. La nuova democrazia progressista, attraverso l'uso dei giornali nazionali e locali, dispone di un "mechanism of developing and exchanging opinions, and of reaching decisions, which is independent of representative assemblies, and which is, or may become, superior to that which it formerly obtained by virtue of occasional popular assemblages"32.

Nel capitolo intitolato Direct vs. Representative Government, Croly sostiene come i padri fondatori, Hamilton e Madison seguendo Montesquieu, fossero pienamente giustificati nel voler tenere separati i tre poteri fondamentali di governo e nel cercare di equilibrarli l'un con l'altro. Il loro errore, se di errore si può parlare all'inizio della storia statunitense, è stato nell'utilizzo dei metodi adottati per stabilire questo equilibrio ${ }^{33}$.

Il sistema costituzionale elaborato a Filadelfia, alla fine del Settecento, scontava il peccato originale di aver affidato compiti legislativi esclusivamente all'aristocrazia naturale americana, la parte del popolo che per ricchezza, capacità e influenza riteneva di potere e dovere rappresentare tutte le altre componenti presenti nella società. Ma il processo di democratizzazione che si stava dispiegando all'inizio del Novecento, e che comportava una piena emancipazione popolare, avrebbe condotto la repubblica americana verso una nuova forma di partecipazione diretta delle masse, la cui conseguenza sarebbe stata la crescita esponenziale di una legislazione sociale progressista e inclusiva.

La democrazia diretta e i vari strumenti politici proposti dai progressisti, dal referendum all'iniziativa legislativa popolare, non dovevano essere concepiti soltanto per migliorare la democrazia rappresentativa e non potevano essere considerati come degli strumenti regressivi. Con lo sviluppo delle moderne comunicazioni di massa "la partecipazione civica sarebbe stata di nuovo possibile su vasta scala, se solo gli americani fossero stati d'accordo nel posporre l'efficienza amministrativa all'educazione politica e morale dei cittadini" ${ }^{34}$.

In maniera ancora più esplicita Walter Weyl esaltava le nuove forme

32 H. Croly, Progressive Democracy, cit., p. 265.

${ }^{33}$ H. Croly, Progressive Democracy, cit., pp. 267-283.

${ }^{34}$ C. LASCH, Il paradiso in terra. Il progresso e la sua critica, Milano, Feltrinelli, 1992, p. 323. 
di democrazia diretta che si andavano affermando soprattutto negli Stati dell'Ovest contro la vecchia democrazia rappresentativa non più in grado di resistere alle sollecitazioni della moderna società industriale. Nel suo volume The New Democracy, Weyl esamina il programma politico della democrazia progressista, con il quale diviene possibile stabilire un controllo completo sul processo di governo. Questa nuova forma di democrazia aveva il compito di demolire il potere della consolidata plutocrazia americana e di stabilire un governo del popolo per il popolo ${ }^{35}$.

Con la democrazia progressista è possibile fissare un controllo del popolo sulla grande industria e sulle lobby politiche finanziate dagli oligopoli industriali. Attraverso le elezioni primarie si realizza, infatti, il controllo democratico dei cittadini sui partiti, mentre attraverso l'istituto del recall è possibile concretizzare il controllo democratico sui rappresentanti eletti. La legislazione diretta da parte del popolo, inoltre, può essere di molto incrementata dall'utilizzo di strumenti quali il referendum e l'iniziativa popolare. Secondo Weyl, il controllo dei cittadini sui partiti politici costituisce l'inizio stesso dell'affermazione della democrazia politica. Non può spettare ai partiti la nomina dei candidati alle elezioni ma è, invece, necessaria una nomina popolare: per scegliere consapevolmente tra due o più candidati gli elettori di un determinato partito devono in precedenza poter scegliere il proprio candidato. Ma una volta eletto il proprio rappresentante, occorreva avere a disposizione, se avesse tradito la fiducia popolare, un metodo abbastanza efficace per poterlo destituire ${ }^{36}$.

Nonostante la lotta degli esponenti progressisti per introdurre in tutti gli Stati e a livello federale le elezioni primarie e il recall, sia Weyl sia Croly si mostravano convinti che questi accorgimenti finissero per assolvere una funzione ottimale soltanto in un periodo di transizione politica, ma sarebbero risultati inutili in un futuro nel quale gli Stati Uniti fossero riusciti ad adottare un'efficace forma di democrazia di-

35 W. E. WeyL, The New Democracy: An Essay on Certain Political and Economic Tendencies in the United States, New York, Macmillan, 1912.

${ }^{36}$ L'istituto del recall, in effetti nel 1912, era già in uso in molte città americane e prevedeva che se almeno il $25 \%$ degli elettori avesse firmato una petizione per destituire l'eletto si sarebbero svolte delle elezioni speciali, nelle quali occorreva che il rappresentante per rimanere in carica ottenesse almeno la metà più uno dei voti. 
retta. A quel punto due sarebbero stati gli istituti complementari della legislazione diretta: il referendum e l'iniziativa popolare. Con il referendum si consente al popolo di porre il veto alle leggi che non desidera, mentre con l'iniziativa legislativa di emanare le leggi che desidera ${ }^{37}$. Il principio fondamentale che ispira l'introduzione del referendum nel sistema politico democratico consiste nella circostanza che ai cittadini viene data la possibilità di esprimere il proprio parere su tutte le tematiche d'importanza fondamentale in campo politico e sociale. "Il referendum dà una voce al Dio muto, Demos. Insieme con l'iniziativa legislativa popolare, esso rappresenta la risposta positiva o negativa del popolo a una determinata domanda, formulata dai legislatori o dal popolo stesso. Esso esprime il potere dei cittadini di proporre leggi ed emendamenti, e di confermare o annullare qualsiasi azione legislativa"38. In queste circostanze, pertanto, il referendum rappresenta il definitivo appello al popolo.

L'adozione del referendum e dell'iniziativa legislativa popolare avrebbe avuto come conseguenza di limitare la capacità di decisione dei legislatori eletti, trasformandoli da rappresentanti a semplici delegati. L'America, riteneva Weyl, stava cercando la cura per superare la democrazia rappresentativa apparente così da poter giungere alla realizzazione di una democrazia diretta effettiva. Tutto ciò sarebbe stato possibile soltanto attraverso la radicale revisione della Costituzione da parte del popolo americano. In un'autentica democrazia politica, il popolo dovrà essere realmente sovrano e lo scopo stesso del regime democratico consisterà, pertanto, nel raggiungimento del benessere sociale. Secondo Weyl, l'obiettivo finale della forma di governo democratica consisteva nello sviluppo fisico, intellettuale e morale dei milioni di cittadini che formavano una nazione. L'avanzamento dei ceti medi e delle masse popolari, determinato dalle riforme progressiste, avrebbe provocato grandi vantaggi in campo politico e industriale alla società americana nel suo complesso ${ }^{39}$.

${ }^{37} \mathrm{O}$. BARIÉ (a cura), Il pensiero politico nell'età di Wilson, cit., pp. 127-28.

${ }^{38}$ O. BARIÉ (a cura), Il pensiero politico nell'età di Wilson, cit., p. 128.

${ }^{39}$ Cfr. C. Forcey, The Crossroads of Liberalism. Croly, Weyl, Lippmann and the Progressive Era 1900-1925, New York, Oxford University Press, 1961. Vedi, anche, J.T. KLoPPENBERG, Uncertain Victory. Social Democracy and Progressivism in European and American Thought, 1870-1920, New York, Oxford University Press, 1986. 
Il pensiero politico di alcuni importanti progressisti americani di inizio Novecento, quali Croly e Weyl, può essere interpretato, pertanto, come una critica alla scienza politica dei Padri Fondatori; costoro volevano rappresentare un nuovo tipo di cittadini e di democratici. L'obiettivo fondamentale da raggiungere era quello di superare la democrazia rappresentativa per giungere alla democrazia diretta, nella quale il popolo americano sarebbe riuscito a ridimensionare la stantia Costituzione federale e una Corte suprema ancora troppo rivolta a difendere le istanze conservatrici delle grandi lobby economiche.

\begin{abstract}
According to the leading figures of the progressive mouvement, the fundamental task of American democracy was to stimulate greater participation of the middle class in the process of government. At the beginning of the twentieth century, it was necessary to recognize that federal institutions were playing an active role in the guarantee of fundamental rights and freedoms of citizens, against strong inequities in American society. The democratic process that was
\end{abstract}

developing in America would lead the country towards a new form of direct participation of the masses, whose consequence would be the affirmation of a progressive social legislation. The solutions for direct democracy, from the referendum to popular initiative, according to the progressives, would improve representative democracy and established a citizens' control over the process of government. A government of the people for the people. 